\title{
Medial Plantar Artery
}

National Cancer Institute

\section{Source}

National Cancer Institute. Medial Plantar Artery. NCI Thesaurus. Code C52968.

One of the terminal branches of the posterior tibial artery that runs along the inside of the foot to supply the muscles of the sole. 